\title{
Risk of Fractures Associated with Dipeptidyl Peptidase- 4 Inhibitor Treatment: A Systematic Review and Meta- Analysis of Randomized Controlled Trials
}

\author{
Qing Chen · Ting Liu • Haonan Zhou • Huawei Peng • \\ Caifeng Yan (D)
}

Received: June 14, 2019 / Published online: July 26, 2019

(C) The Author(s) 2019

\begin{abstract}
Introduction: More and more studies suggest that type 2 diabetes mellitus (T2DM) can lead to an increased fracture risk. Some previous clinical studies and experimental data have shown that some antidiabetic drugs can increase or decrease the incidence of fractures.

Methods: We searched Medline, Embase, Cochrane Library, and the ClinicalTrials.gov website (https://www.clinicaltrials.gov) for published or unpublished randomized controlled trials (RCTs) from inception through 2 December 2018 to compare the effects of dipeptidyl peptidase-4 (DDP-4) inhibitors with active control drugs or placebo in T2DM patients. All RCTs had a duration of at least 12 weeks, and the ultimate measure was
\end{abstract}

Enhanced Digital Features To view enhanced digital features for this article go to https://doi.org/10.6084/ m9.figshare.8846225.

Electronic Supplementary Material The online version of this article (https://doi.org/10.1007/s13300019-0668-5) contains supplementary material, which is available to authorized users.

Q. Chen · T. Liu · H. Zhou · H. Peng · C. Yan ( $ه)$ Department of Endocrinology, Northern Jiangsu People's Hospital, Yangzhou 225001, China e-mail: 778739065@qq.com whether a fracture occurs or not. We calculated odds ratios and their 95\% confidence intervals by the fixed effect Mantel-Haenszel model. Publication bias was investigated firstly through visual observation of funnel plot asymmetry and then through Begg's test or Egger's test. The Cochrane bias risk tools were used to assess the quality of included studies.

Results: Eighty-seven eligible RCTs were included in this study. Of 93,772 participants, 49,270 patients received therapy and 44,502 were control patients. Five kinds of DDP-4 inhibitors were included: sitagliptin, saxagliptin, alogliptin, linagliptin and vildagliptin. There were 676 fractures in the DDP-4 inhibitor treatment group and 646 in the control group. The median average glycosylated hemoglobin level was $8.2 \%$. DDP-4 inhibitor treatment did not seem to influence the fracture risk, no matter whether compared with placebo or active comparators in T2DM patients (Mantel-Haenszel odds ratio $(\mathrm{MH}-\mathrm{OR})=1.01,95 \%$ CI 0.90-1.12, $P=0.92)$. After three subgroup analyses which were defined by drug type, control regimen and duration, the results were still stable.

Conclusion: This systematic review and metaanalysis shows that DDP-4 inhibitors do not affect the fracture risk when compared with antidiabetic drugs or placebo in T2DM patients.

Keywords: Dipeptidyl peptidase- 4 inhibitors; Fracture; Meta-analysis; Type 2 diabetes mellitus 


\section{INTRODUCTION}

Diabetes affects an estimated 6-8\% of the population worldwide [2] and more than $90 \%$ of patients with diabetes are classified as having type 2 diabetes mellitus (T2DM). As we all know, T2DM is a universal disease caused by lifestyle and/or genetic factors; obese patients and the elderly are the predominant groups affected.

More and more studies suggest that type 2 diabetes can lead to an increased fracture risk $[2,14,31]$. Although there is no clear pathogenic mechanism, some possible mechanisms have been found, such as changes in bone mineral density [2], impaired skeletal quality and strength, or the effects of comorbidities such as diabetic macrovascular and microvascular complications [28]. Although bone fractures are not considered to be the main complications of T2DM in the traditional sense, bone fractures in diabetic patients can lead to decreased blood glucose control, physical disability, and declining overall life quality [11].

Some previous clinical studies and experimental data have shown that some antidiabetic drugs can increase or decrease the incidence of fractures [1, 25]. For instance, thiazolidinediones can hamper osteoblastogenesis and increase the bone loss and fracture risk; the effect on postmenopausal women with T2DM is particularly significant [16]. Conversely, the use of sulfonylureas and metformin can reduce fracture risk and they have a positive impact on skeletal health [1]. It is worth mentioning that although insulin treatment has no significant effect on bone mineral density [17], insulin treatment also increases the risk of fracture [29].

Dipeptidyl peptidase-4 (DDP-4) inhibitors are a class of antidiabetic drugs which can increase insulin levels and improve glycemic control by increasing the levels of glucagon-like peptide 1 (GLP-1) and glucose-dependent insulinotropic polypeptide (GIP), and they do not lead to increased weight or increased hypoglycaemic risk in diabetic patients [5].

In order to understand the specific connections between DDP-4 inhibitors and the risk of fractures, more and more research has been conducted. However, the results obtained are contradictory. A previous meta-analysis and systematic review of randomized controlled trials (RCTs) showed that treatment with DPP-4 inhibitors can decrease fracture risk by $40 \%$ in T2DM patients when compared with active control therapies or placebo [23]. In contrast, the results of another meta-analysis and systematic review suggested that there was no obvious correlation between fracture risk and DPP-4 inhibitor treatment when compared with active comparators or placebo [21]. According to a previous cohort study, DPP-4 inhibitor therapy did not affect the risk of fracture compared with non-insulin hypoglycemic drug users and control groups [8]. A post hoc pooled analysis showed that after the summary of 20 RCTs, the incidence of fractures with saxagliptin was higher than that in the control group [13]. In animal model tests, sitagliptin treatment may reduce bone resorption, further increase bone strength, and reduce bone loss in diabetic rats [9].

In previous systematic reviews, few studies directly investigated the relationship between DPP-4 inhibitor treatment and fracture. Bone fractures are generally considered as adverse events in research rather than as major findings. In recent years, many new powerful RCTs have investigated DPP-4 inhibitors. Therefore, we summarized all RCTs of DPP-4 inhibitors compared with other drugs for diabetes mellitus or placebo. The aim of this research is to obtain a meta-analysis and systematic review to ascertain whether the treatment of DPP-4 inhibitors is related to the occurrence of fracture in T2DM patients or not.

\section{METHODS}

\section{Data Sources and Search Strategy}

Two investigators (QC and TL) conducted extensive searches of Medline, Embase, and Cochrane Central Register of Controlled Trials independently, and a senior investigator (HZ) resolved any conflicts. We searched the electronic database to collect data from all human 
RCTs up to 2 December 2018. By searching the ClinicalTrials.gov website (https://www. clinicaltrials.gov), we identified trials completed but not yet published. The following Medline's search strategy is also applicable to other electronic databases:

1. Exp (dipeptidyl-peptidase IV inhibitors).

2. (Dipeptidyl-peptidase IV or gliptins or DPP-4 or dipeptidyl peptidase 4).tw.

3. (Sitagliptin phosphate or saxagliptin or linagliptin or vildagliptin or alogliptin or anagliptin or trelagliptin).tw.

4. 1 or 2 or 3 .

5. (Diabetes mellitus, type 2).tw.

6. 4 and 5 .

7. Randomized controlled trial.pt.

8. Randomized.tiab.

9. Randomly.tiab.

10. Placebo.tiab.

11. Trial.tiab.

12. Controlled clinical trial.pt.

13. Drug therapy.sh.

14. Groups.tiab.

15. 7 or 8 or 9 or 10 or 11 or 12 or 13 or 14 .

16. Exp animals.mh not humans.mh.

17. 15 not 16 .

18. 6 and 17.

\section{Study Selection}

The trials that satisfied the following conditions were included in our study: (1) only RCTs in T2DM patients; (2) with a duration greater than or equal to 12 weeks; (3) DDP-4 inhibitors as interventions, including sitagliptin, saxagliptin, vildagliptin, alogliptin, linagliptin, trelagliptin, and anagliptin; (4) comparing the effects of DDP-4 inhibitors with comparators or placebo; and (5) data on fracture occurrence are available. Trials in which both groups had zero incidents were eliminated.

\section{Data Extraction Content and Quality Assessment}

We extracted the following relevant information from RCTs that met inclusion criteria: name of the first author, sample size, publication year, duration of the trial, types of DPP-4 inhibitors and comparators, mean age, glycosylated hemoglobin (HbA1c) level (\%), and reported results (number of fracture events per treatment group). Two investigators (QC and TL) performed data collection independently, the results of which were kept in duplicate. A senior investigator (HZ) addressed any conflicts. The Cochrane bias risk tools were used to assess the quality of included studies [12] and included the following seven items: (1) randomization, (2) description of allocation, (3) blinding of participants/personnel, (4) blinding of outcome assessment, (5) incomplete outcome data, (6) selective reporting, and (7) other sources of bias.

\section{Data Analysis}

The meta-analysis was reported in strict accordance with the PRISMA guideline [26]. The major observation result was the presence or absence of fracture, regardless of fracture sites. We calculated odds ratios (ORs) and their 95\% confidence intervals by the fixed effect Mantel-Haenszel model. $I^{2}$ statistics was used to assess heterogeneity. An $I^{2}$ value greater than or equal to $50 \%$ was considered to indicate significant heterogeneity among the trials [4]. According to the results of Cochrane's $Q$ test, the choice of fixed or random effect model was determined. In order to exclude specific studies that could alter current research results, sensitivity or subgroup analysis was conducted. To inspect the impact of specific research characteristics (such as mean age and glycosylated hemoglobin) on risk effects, we carried out meta-regression analyses. Publication bias was investigated firstly through visual observation of funnel plot asymmetry and then through Begg's test or Egger's test. Statistical analysis of the data was performed by using STATA 14.0 (STATA Corp, TX, USA) and RevMan Version 5.3 (Cochrane Collaboration, Oxford, UK). The GRADE system was used to assess the quality of evidence by outcome [3].

\section{Compliance with Ethics Guidelines}

This article is based on previously conducted studies and does not contain any studies with human participants or animals performed by any of the authors. 


\section{RESULTS}

The initial database searches identified 2858 unique titles and abstract. After duplicate article records were deleted, 1651 records were retained. The analysis eventually included 87 RCTs, among which 67 clinical trials were obtained from journals and 20 clinical trials were from the ClinicalTrials.gov website (https://www.clinicaltrials.gov). The flowchart of the studies included is shown in Fig. 1.

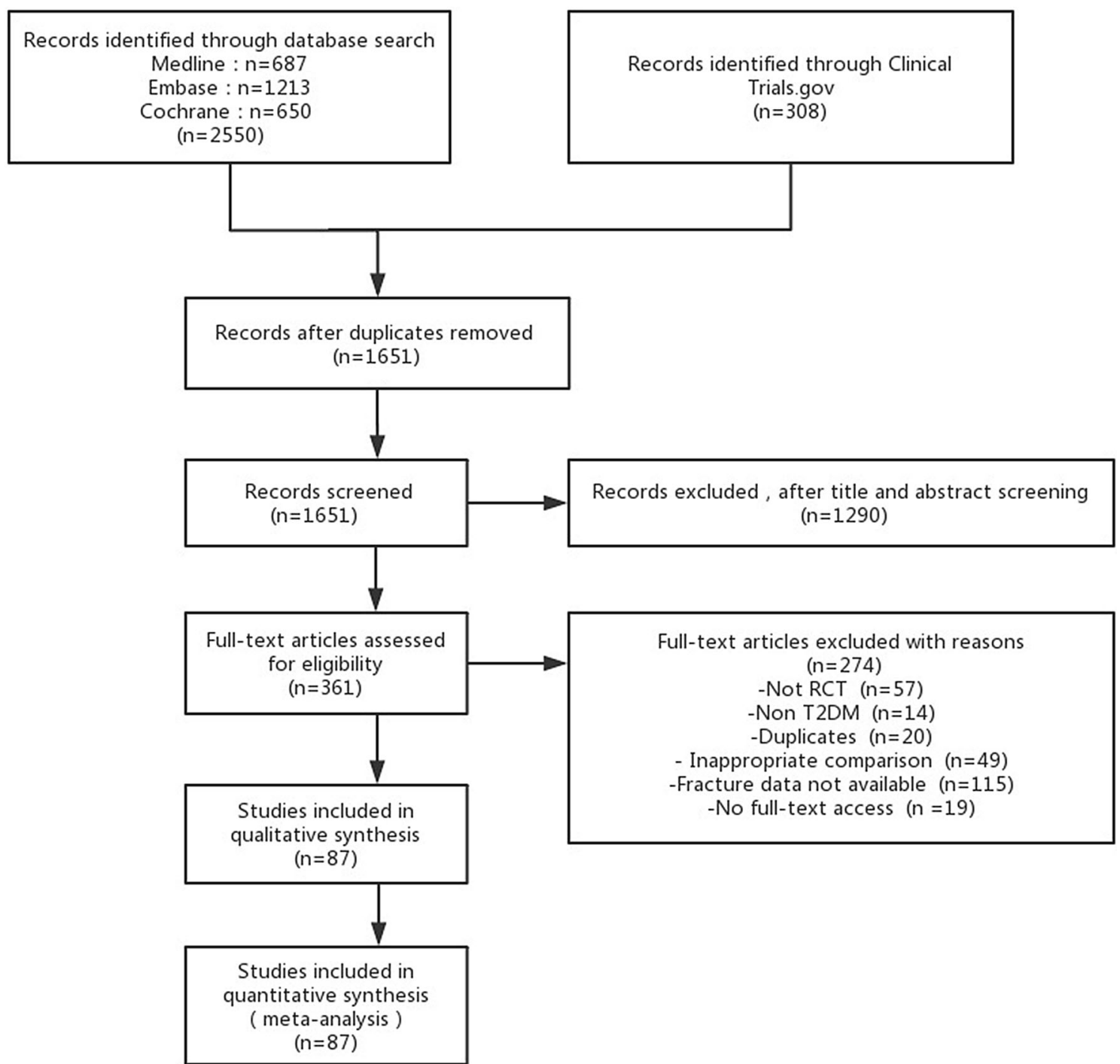

\section{Characteristics of Research Included in the Study}

The characteristics of research included in the study are presented in Table 1 . This analysis included 93,772 participants, including 49,270 in the DDP-4 inhibitor treatment group and the others were in the control group. The total number of fracture incidents was 1322, arising from 676 participants in the DDP-4 inhibitor treatment group and 646 participants in the control group. The DDP-4 inhibitors included in all 87 trials were as follows: sitagliptin in 45 , saxagliptin in 15 , linagliptin in 15 , alogliptin in seven, and vildagliptin in five. A total of 28 


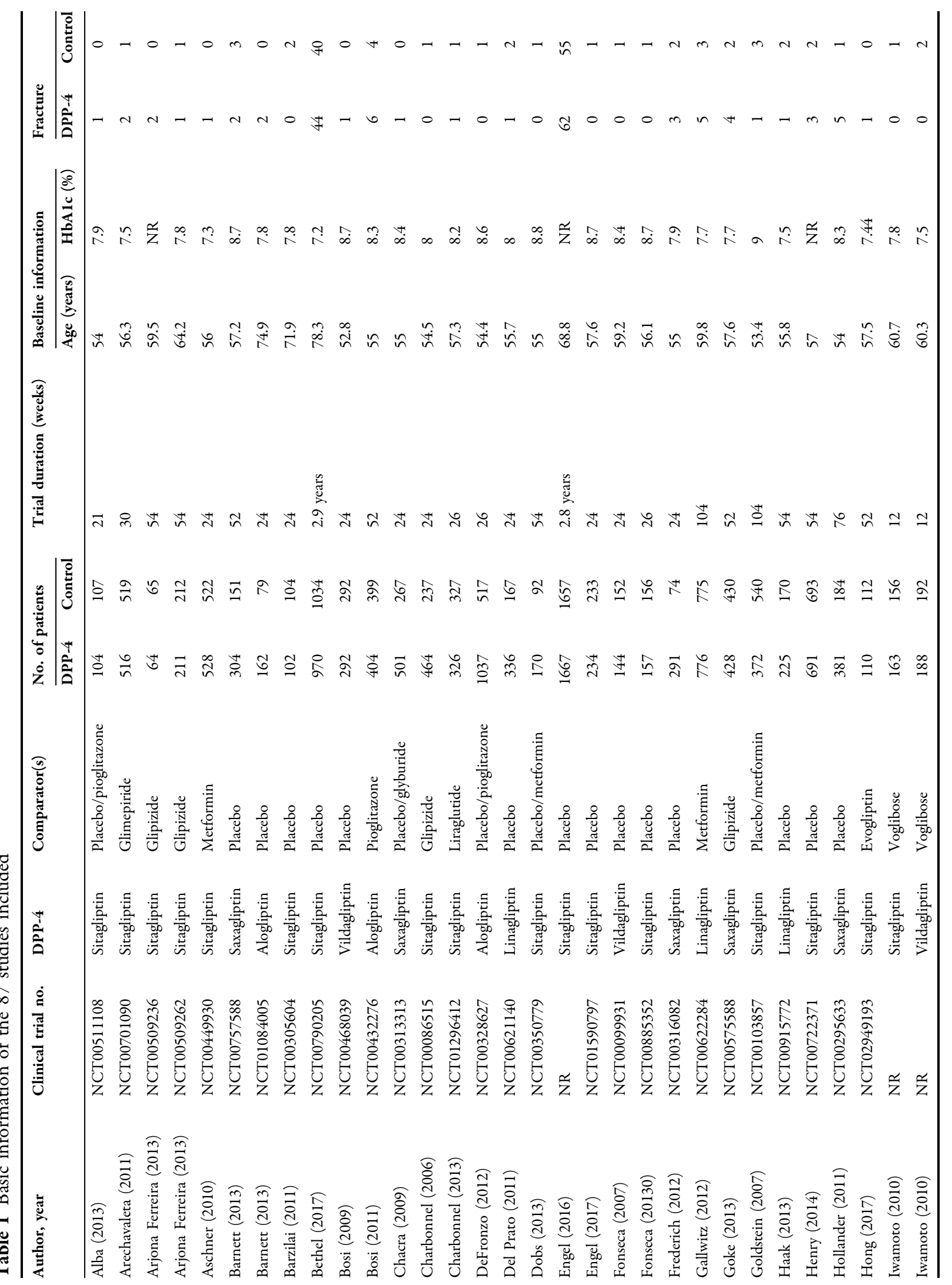




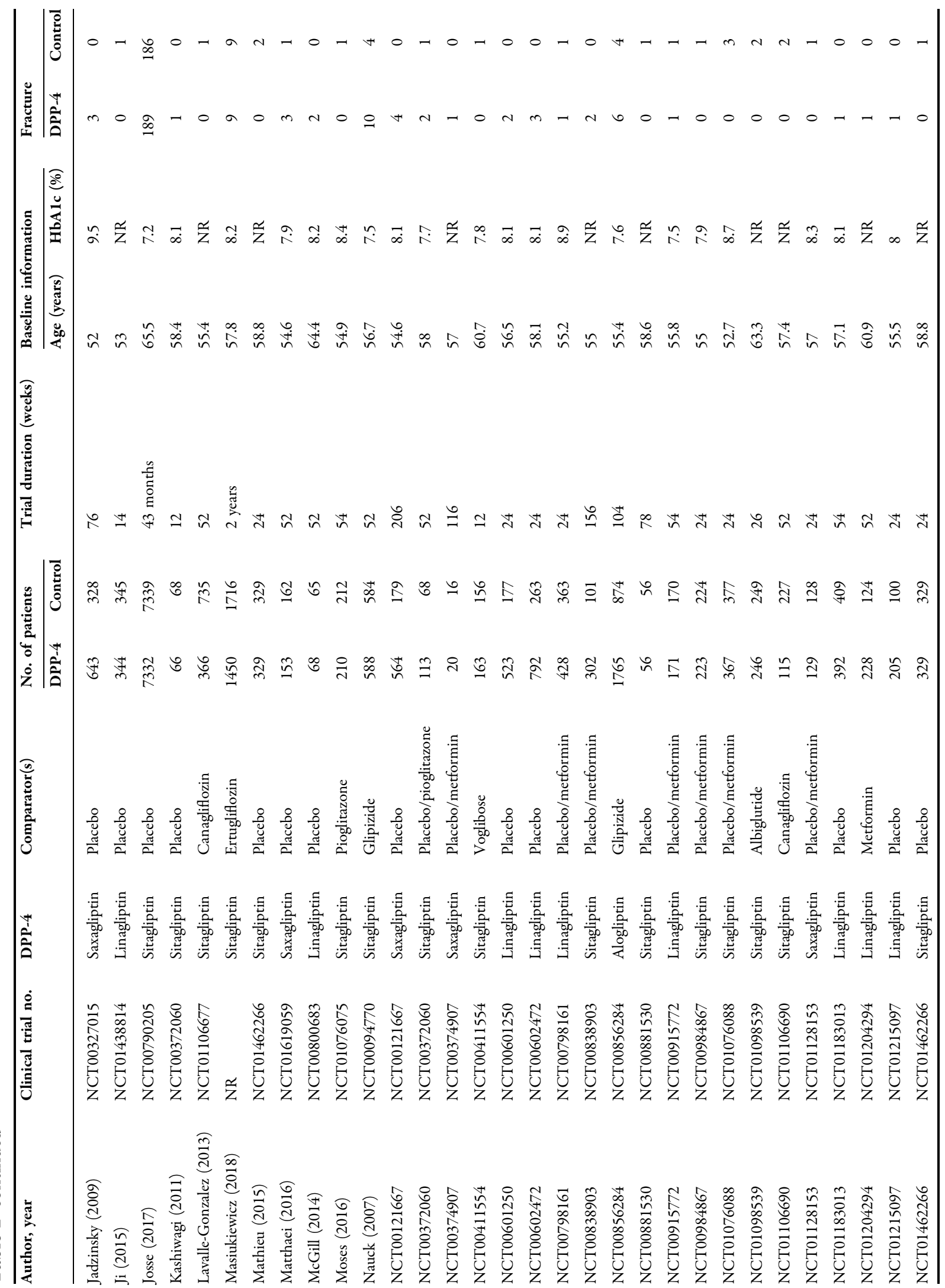




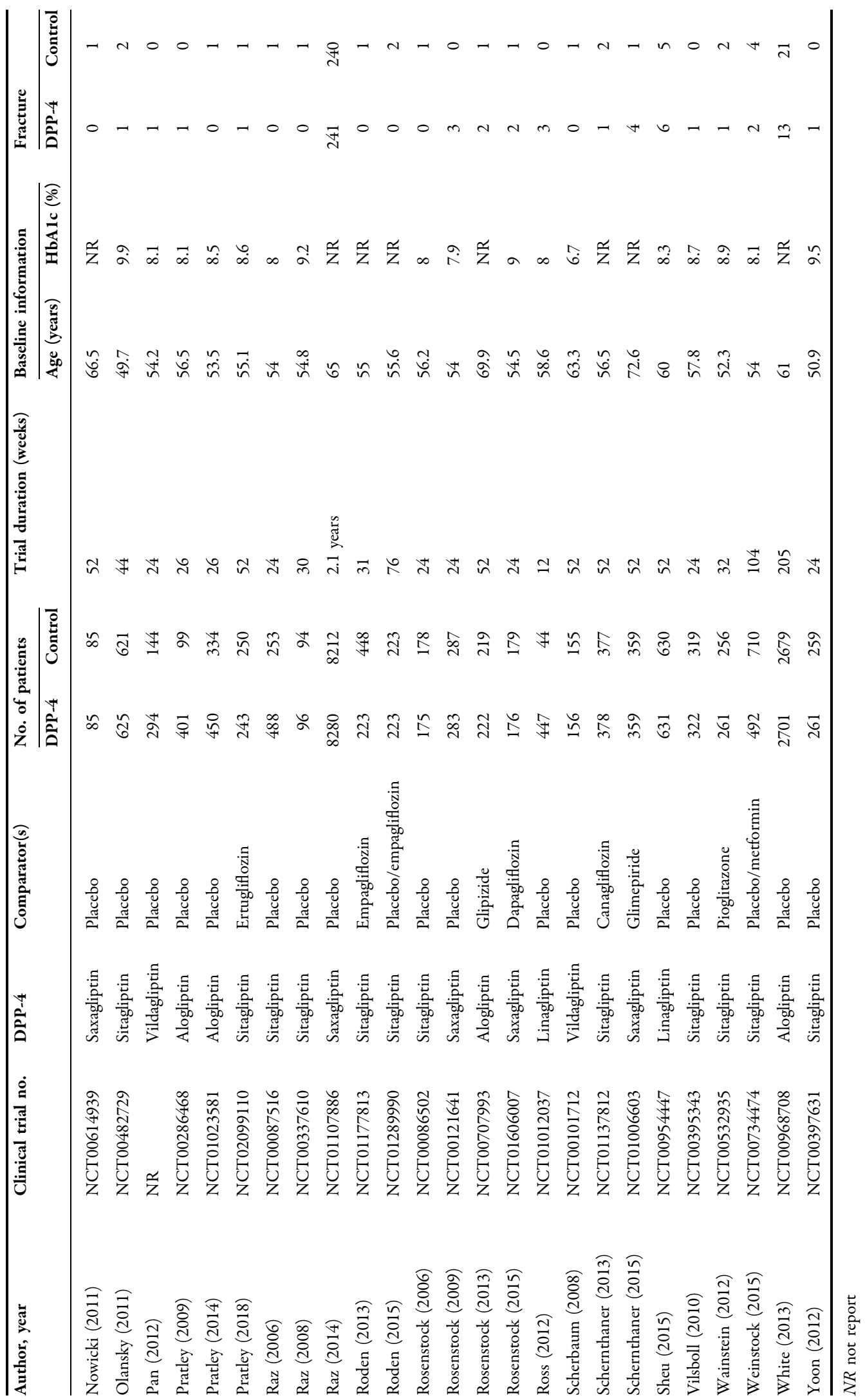


studies and 44 studies, including 22,082 patients and 63,135 patients, were used to compare DPP-4 inhibitors with active comparators and placebo, respectively, while 15 trials including 8555 patients compared DPP-4 inhibitors with active comparators and placebo simultaneously. Five traditional antidiabetic drugs were included as active comparators: sulfonylureas, thiazolidinediones, metformin, GLP-1 receptor agonists, and SGLT2 inhibitors. Among the studies included, the earliest one was published in 2006, and the latest one was published in 2018. The main population in the study was middle-aged and elderly people; the average age of the study population was 49.7-78.3 years. Follow-up time of the study ranged from 12 to 206 weeks. We used 52 weeks as the cutoff point for the duration of treatment; 44 RCTs lasted less than it and 43 RCTs lasted longer.

\section{Evaluation of Quality}

Table S1 and Fig. S1 (supplementary material) summarize the quality assessment of 87 studies included in this analysis. We evaluated 87 studies one by one using the Cochrane bias risk tools and found that nine studies did not mention the method used for randomization. The methods used to describe assignment and blinding method for outcome assessment were unclear in 19 and 44 studies, respectively. Almost all the studies described the method for handling incomplete outcome data, blinding of participants and personnel, and selective reporting. Overall, the bias risk in research was considered relatively low.

\section{Meta-Analysis: Risk of Fractures with DDP-4 Inhibitor Treatment}

In order to understand the incidence of fracture related to DPP-4 inhibitor treatment, we conducted statistical analyses. Overall, DPP-4 inhibitor therapy did not influence the fracture risk, no matter whether compared with placebo or active comparators in T2DM patients $(\mathrm{MH}-$ $\mathrm{OR}=1.01,95 \%$ CI $0.90-1.12, P=0.92$, Fig. 2 ). Heterogeneity was not observed $\left(I^{2}=0 \%\right.$, $P=1.00)$. Through the GRADE system, we believe that the quality of evidence was moderate. The quality of evidence is summarized in Table S2 (supplementary material).

\section{Sensitivity Analysis}

No low-quality studies were found in sensitivity analysis, and the results were approximately similar to those of the major analysis (Fig. S2, supplementary material).

\section{Subgroup Analysis}

\section{According to Control Regimen}

The forest plot in Fig. S3 (supplementary material) shows a subgroup analysis which compared DPP-4 inhibitors to the control regimen. A total of 30,637 participants in trials were compared using active comparators, the $\mathrm{MH}-\mathrm{OR}$ was 1.04 (95\% CI $0.74-1.46, P=0.81$ ) in trials vs. active comparators, and fractures occurred in 72 cases and 68 cases in the DDP- 4 inhibitor treatment group and the control group, respectively. The $I^{2}$ value obtained was equal to $0.0 \%$ and heterogeneity was not observed. A total of 71,690 participants in trials were compared using placebo, the $\mathrm{MH}-\mathrm{OR}$ was 0.99 (95\% CI $0.88-1.11, P=0.88)$ in trials vs. placebo, and fractures occurred in 616 cases and 597 cases in the DDP-4 inhibitor treatment group and the control group, respectively. The obtained $I^{2}$ was $0.0 \%$ and heterogeneity was not observed. The results of subgroup analysis indicated that the effect of DPP-4 inhibitors was not significantly different between different control regimens $(P=0.79)$. Through the GRADE system, we believe that the quality of evidence was moderate. The quality of evidence is summarized in Table S2 (supplementary material).

\section{According to Type of DPP-4 Inhibitor}

The forest plot in Fig. S4 (supplementary material) shows a subgroup analysis which compared the effects of different types of DDP-4 inhibitors on fracture risk. In a total of 46,415 participants in 45 trials using sitagliptin, MH-OR for sitagliptin was 1.02 (95\% CI 0.88-1.19, $P=0.79)$. Among them, 340 participants and 342 participants were in the DDP-4 inhibitor treatment 


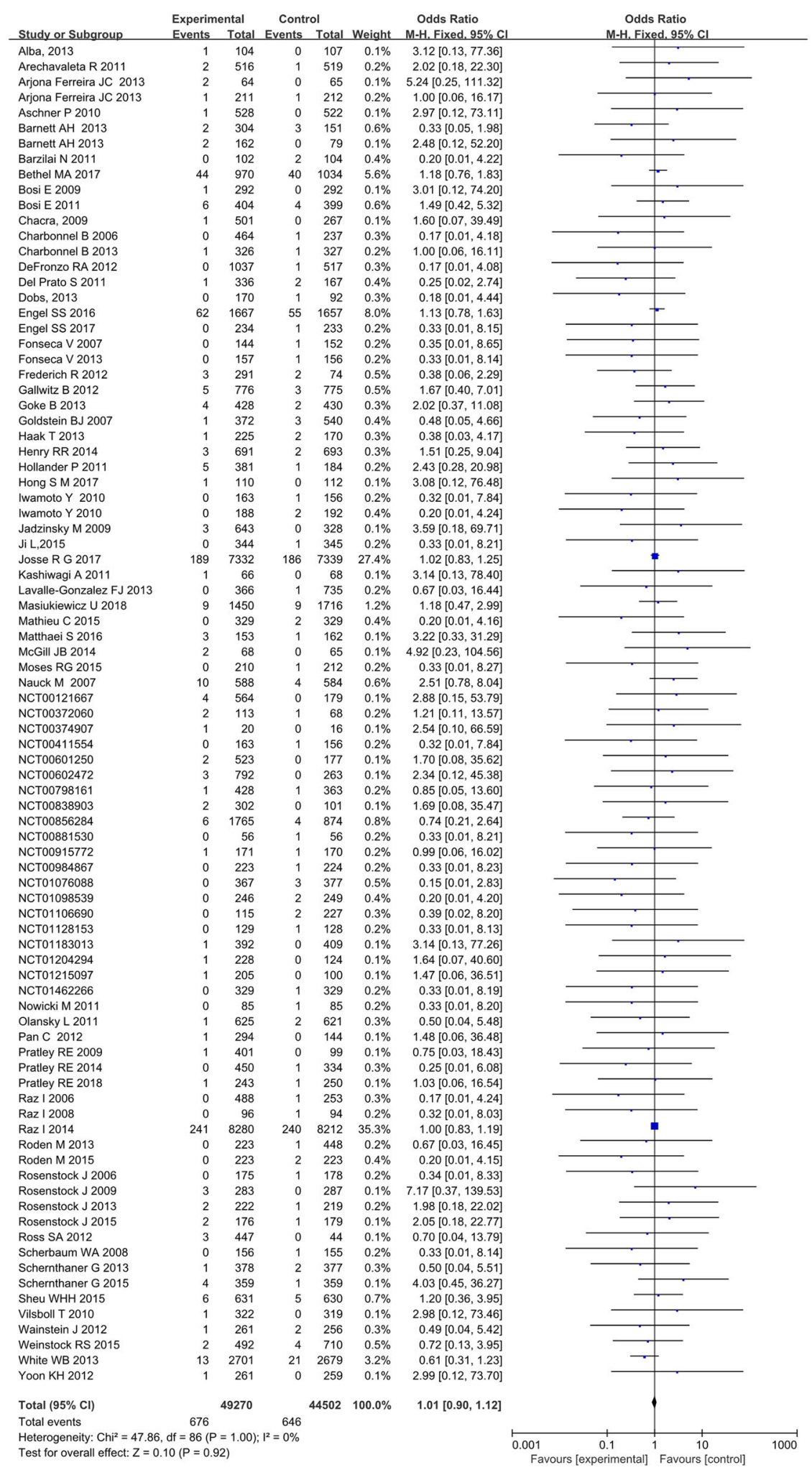

Fig. 2 Risk of bone fractures associated with dipeptidyl peptidase- 4 inhibitor treatment 
group and the control group, respectively. In a total of 23,638 participants in 15 trials using saxagliptin, MH-OR for saxagliptin was 1.02 (95\% CI $0.86-1.22, P=0.81$ ), including 276 participants and 253 participants in the saxagliptin group and the control group, respectively. In a total of 9609 participants in 15 trials using linagliptin, MH-OR for linagliptin was 1.16 (95\% CI $0.64-2.13, P=0.62$ ), including 30 participants and 15 participants in the linagliptin group and the control group, respectively. In a total of 12,101 participants in seven trials using alogliptin, $\mathrm{MH}-\mathrm{OR}$ for alogliptin was 0.73 (95\% CI $0.44-1.22, P=0.23$ ), including 28 participants and 32 participants in the alogliptin group and the control group, respectively. In a total of 2009 participants in five trials using vildagliptin, $\mathrm{MH}-\mathrm{OR}$ for vildagliptin was 0.62 (95\% CI $0.15-2.56, P=0.51$ ), including two participants and four participants in the vildagliptin group and the control group, respectively. The $I^{2}$ value was equal to $0.0 \%$ in all drug types, indicating no presence of heterogeneity. Overall, between DPP-4 inhibitors of different drug types there was no significant statistical difference in fracture risk after subgroup analysis $(P=0.70)$. Through the GRADE system, we believe that the quality of evidence was moderate. The quality of evidence is summarized in Table S2 (supplementary material).

\section{According to Duration}

The forest plot in Fig. S5 (supplementary material) shows a subgroup analysis which compared the effects of different length of follow-up on fracture risk. In a total of 24,915 participants with a length of follow-up of less than 52 weeks, MH-OR for follow-up of less than 52 weeks was 0.65 (95\% CI 0.42-1.01, $P=0.06$ ), including 35 participants and 39 participants in the DDP-4 inhibitor treatment group and the control group, respectively. In a total of 16,334 participants in trials with a length of follow-up of at least 52 weeks and less than 104 weeks, MH-OR for that was 1.28 (95\% CI 0.85-1.91, $P=0.24)$, including 62 participants and 42 participants in the DDP-4 inhibitor treatment group and the control group, respectively. In a total of 31,326 participants in trials with a length of follow-up of at least 104 weeks and less than 156 weeks,
MH-OR for that was 1.04 (95\% CI $0.90-1.20$, $P=0.62$ ), including 371 participants and 358 participants in the DDP-4 inhibitor treatment group and the control group, respectively. In a total of 21,197 participants in trials with a length of follow-up of at least 156 weeks, $\mathrm{MH}$ OR for that was 0.98 (95\% CI 0.81-1.20, $P=0.87$ ), including 208 participants and 207 participants in the DDP-4 inhibitor treatment group and the control group, respectively. The obtained $I^{2}$ was $0.0 \%$ and heterogeneity was not observed. Subgroup analysis based on the duration of DPP-4 inhibitor therapy showed no significant difference in fracture risk $(P=0.90)$. The test for subgroup differences $(P=0.15$, $I^{2}=43.1 \%$ ) refers to the heterogeneity between subgroups. It is considered that the heterogeneity is not enough to influence the results. The evidence quality was moderate (Table S2, supplementary material).

\section{Meta-Regression}

To inspect the effect of specific research characteristics (mean age and glycosylated hemoglobin) on fracture risk, we also performed meta-regression analyses. We observed that the risk of fracture did not change with mean age $(P=0.222)$ and glycosylated hemoglobin $(P=0.406)$ level and no statistically significant difference effect was noted (Fig. S6, supplementary material).

\section{Funnel Plot and Publication Bias}

The funnel plot in Fig. 3 does not show any sign of publication bias by visual inspection, and Begg's test $(P=0.077)$ and Egger's test $(P=0.170)$ indicated no major publication bias.

\section{DISCUSSION}

Bone fractures in diabetic patients can lead to decreased blood glucose control, physical disability, and declining overall life quality, so fractures are receiving increasing attention and have become one of the important endpoints of clinical trials. On the basis of previous studies, we believe that the relationship between DDP-4 


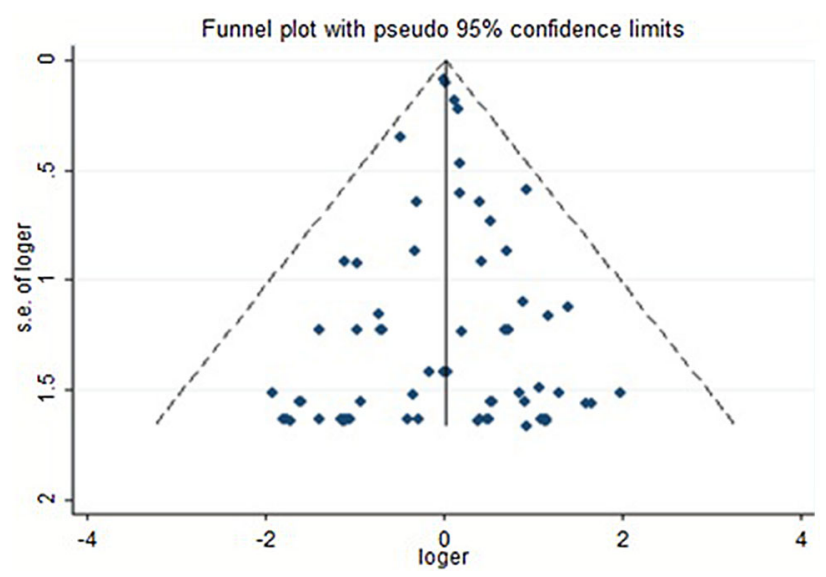

Fig. 3 Funnel plot

inhibitors and fracture risk in T2DM patients is unclear. And in recent years, many new powerful RCTs have been published to investigate DPP-4 inhibitors, so we performed a metaanalysis and systematic review to ascertain whether the treatment with DPP-4 inhibitors is related to the occurrence of fracture in T2DM patients or not. Analysis of data from 87 RCTs showed that DPP-4 inhibitors did not affect the fracture risk when compared with antidiabetic drugs or placebo in T2DM patients.

The quality of the studies was evaluated using the Cochrane bias risk tool. From Table S1 and Fig. S1 (supplementary material), it can be concluded that the bias risk in RCTs is relatively low, the design of the study is reasonable, and the intensity of evidence is high. The forest plots of the meta-analysis and three subgroup analyses (drug types, control regimen, and duration) showed an $I^{2}$ value of $0.0 \%$, indicating no statistical heterogeneity. This means that it is not the heterogeneity of the research itself, but any variation in the research may be attributed to changes in the independent variables [4]. According to the GRADE system the evidence quality is moderate. Consistency of data analysis results between studies can provide confidence in the application of the results. The analysis results have good internal credibility. Moreover, the sample size of the study was large and more than 90,000 people participated; the randomized clinical trial data from multiple countries is representative, reflecting the external validity of the study.
Our results were consistent with a retrospective cohort study of 216,816 participants treated with DPP- 4 inhibitors for 1.3 years. The results suggested that DPP-4 inhibitor therapy did not affect the fracture risk compared with non-insulin hypoglycemic drug users and control groups [8]. The results of another analysis previously done by Monami et al. are contrary to ours [23]. They concluded that DPP-4 inhibitor users have a $40 \%$ reduction in the risk of fracture. Their study included a total of 21,055 participants in 28 clinical trials, including 11,880 using DPP-4 inhibitors and 9175 using comparators; the mean treatment time was 35 weeks. We collected data from 87 randomized trials involving a total of 93,772 participants, with an average treatment time of 52 weeks, and the number of patients was approximately four times higher than that included in Monami et al.'s study. The treatment time is also relatively long. In addition, we conducted three subgroup analyses which were defined by drug types, control regimen, and duration to explore sources of heterogeneity and the results remained robust. Therefore, we believe that the difference in research results may be attributed to the relatively few participants in the research and the short observation time.

Of the 87 studies included, two were large RCTs, one of which was published by Josse et al. in 2017, which included 14,671 participants treated with sitagliptin and placebo for 43 months [15]. The other RCT had a total of 
16,492 participants and was completed by Raz et al. in 2014 who set a control for saxagliptin vs. placebo for a 2.1-year clinical trial [27]. The results showed that the incidence of fracture in DPP-4 inhibitor users was approximately the same as that in placebo users. Our results were influenced to some extent by these two studies $(N=31,163)$, so we performed a sensitivity analysis. However, after these two tests were omitted, the results were still robust.

DPP-4 inhibitors and GLP-1 receptor agonists have similar hypoglycemic mechanisms. Both drug types lower blood glucose by increasing circulating levels of GLP-1. Previous research has suggested that GLP-1 may have a beneficial effect on bone metabolism [24, 32]. Type 2 diabetic rats given GLP-1 can continuously promote bone formation and thus exert bone anabolism [24]. Increased bone resorption of osteoclasts in mice with GLP-1 receptor knockout results in a decrease in cortical bone mass and an increase in bone fragility [32]. In in vitro mice experiments, the cortical thickness and bone outer diameter of mice lacking the GLP-1 receptor were significantly reduced, reflecting a decrease in bone strength, indicating that GLP1 is beneficial to bone strength and quality [18]. However, the results of another analysis previously done by Mabilleau et al. did not provide significant evidence that fracture risk reduced in participants treated with GLP-1 receptor agonists [19]. The result of another meta-analysis is interesting: liraglutide treatment significantly reduced the fracture incidence in T2DM patients; however, fracture risk increased in participants treated with exenatide [30]. A recent analysis suggests that exenatide is the safest option and has the lowest risk of fracture compared with other GLP-1 receptor agonists [33]. Different from GLP-1 receptor agonists, DPP-4 inhibitors can increase GIP levels. A previous 5-day crossover study of patients with type 1 diabetes suggested that short-term GIP infusion could significantly reduce bone resorption and improve bone metabolism [7]. In animal models, GIP was found to enhance glucose-induced insulin secretion and bind to GIP receptor (GIPR) for bone synthesis [10]. The results of another animal experiment supported the positive effects of GIPR on bone quality and strength [22]. A previous study found that GIP reduced osteoclast formation, differentiation, and absorption in ovariectomized mice [20].

After meta-regression of special study characteristics, we believe that age and glycated hemoglobin levels have no effect on our findings. In addition, we consider that other potential reasons such as differences in longterm habits of individuals may have an impact. Differences in previous RCT evaluations indicate that the long-term impacts of DPP-4 inhibitors on fractures are not conclusive.

Although our study only explored whether DPP-4 inhibitors alone could increase the risk of fractures in T2DM patients, in practical clinical work, most T2DM patients are treated with two or more combination hypoglycemic drugs. In a nationwide study of South Korea, DDP-4 inhibitors combined with metformin had a lower incidence of fracture than sulfonylureas combined with metformin. The results showed that DDP-4 inhibitors combined with metformin may have a protective effect on bone metabolism compared with sulfonylureas [6]. We also found research into combined treatment with DDP-4 inhibitors and other hypoglycemic drugs, but results including fracture data were rare. Therefore, it is necessary to strengthen the research on the effect of DDP-4 inhibitors combined with hypoglycemic drugs on fracture in the future.

There are still some limitations in the studies we have included. The mean follow-up time of the RCTs was 52 weeks, which we believe is probably too short to observe the occurrence of the fracture event. Therefore, we performed a subgroup analysis using 52 weeks as the cutoff point for treatment time and found no significant differences in fracture risk at different follow-up times. In most of the trials involving fracture outcomes, fractures were not considered to be the major findings of the trial report and were only recorded as serious adverse events. The results of some of the data were not reported. For example, in some published articles, there is no information on glycated hemoglobin, and the data is labeled as "NR". We acknowledge that these missing data may result in incomplete images of patient baseline characteristics. However, the missing data is not 
related to the main results of this study. In addition, there is no data on racial, gender, and female menopausal status in the initial data included, and the special features included are not comprehensive. Therefore, trials with longer duration of treatment and fractures as the main outcome are needed to explore the relationship between DPP-4 inhibitor use and fracture occurrence.

\section{CONCLUSION}

Although the results of our study indicate that DPP-4 inhibitors show no significant anti-fracture capabilities, this study is still of value to physicians when choosing these drugs as a treatment option. In addition, treatment of diabetic patients with DDP-4 inhibitors, which is thus usually independent of the risk of fracture, can be considered as an advantage worth mentioning compared with drugs such as thiazolidinedione or exenatide, which are known to increase the risk of fracture.

\section{ACKNOWLEDGEMENTS}

Funding. The Social Development Foundation of Yangzhou City of China (YZ2016061) funded this study and the journal's Rapid Service Fee.

Authorship. All named authors meet the International Committee of Medical Journal Editors (ICMJE) criteria for authorship for this article, take responsibility for the integrity of the work as a whole, and have given their approval for this version to be published.

Disclosures. Qing Chen, Huawei Peng, Haonan Zhou, Ting Liu, and Caifeng Yan have nothing to disclose.

Compliance with Ethics Guidelines. This article is based on previously conducted studies and does not contain any studies with human participants or animals performed by any of the authors.
Data Availability. All data generated or analyzed during this study are included in this published article/as supplementary information files.

Open Access. This article is distributed under the terms of the Creative Commons Attribution-NonCommercial 4.0 International License (http://creativecommons.org/licenses/ by-nc/4.0/), which permits any noncommercial use, distribution, and reproduction in any medium, provided you give appropriate credit to the original author(s) and the source, provide a link to the Creative Commons license, and indicate if changes were made.

\section{REFERENCES}

1. Adil M, Khan RA, Kalam A, et al. Effect of anti-diabetic drugs on bone metabolism: evidence from preclinical and clinical studies. Pharmacol Rep. 2017;69(6):1328-40.

2. Botushanov NP, Orbetzova MM. Bone mineral density and fracture risk in patients with type 1 and type 2 diabetes mellitus. Folia Med. 2009;51(4): $12-7$.

3. Brożek JL, Akl EA, Jaeschke R, et al. Grading quality of evidence and strength of recommendations in clinical practice guidelines: part 1 of 3 . An overview of the GRADE approach and grading quality of evidence about interventions. Allergy. 2010;64(5): 669-77.

4. Chen B, Benedetti A. Quantifying heterogeneity in individual participant data meta-analysis with binary outcomes. Syst Rev. 2017;6(1):243.

5. Chen XW, He ZX, Zhou ZW, et al. Clinical pharmacology of dipeptidyl peptidase- 4 inhibitors indicated for the treatment of type 2 diabetes mellitus. Clin Exp Pharmacol Physiol. 2015;42(10):999-1024.

6. Choi HJ, Park C, Lee YK, et al. Risk of fractures and diabetes medications: a nationwide cohort study. Osteoporos Int. 2016;27(9):2709-15.

7. Christensen MB, Lund A, Calanna S, et al. Glucosedependent insulinotropic polypeptide (GIP) inhibits bone resorption independently of insulin and glycemia. J Clin Endocrinol Metab. 2017;103(1):288-94.

8. Driessen JHM, Van Onzenoort HAW, Henry RMA, et al. Use of dipeptidyl peptidase- 4 inhibitors for type 2 diabetes mellitus and risk of fracture. Bone. 2014;68:124-30. 
9. Glorie L, Behets GJ, Baerts L, et al. DPP4 inhibitor treatment attenuates bone loss and improves mechanical bone strength in male diabetic rats. Am J Physiol Endocrinol Metab. 2014;307(5):E447.

10. Garg G, McGuigan FE, Kumar J, Luthman H, Lyssenko V, Akesson K. Glucose-dependent insulinotropic polypeptide (GIP) and GIP receptor (GIPR) genes: an association analysis of polymorphisms and bone in young and elderly women. Bone Rep. 2016;4:23-27.

11. Hamann C, Kirschner S, Günther KP, et al. Bone, sweet bone-osteoporotic fractures in diabetes mellitus. Nat Rev Endocrinol. 2012;8(5):297-305.

12. Higgins JP, Thompson SG, Deeks JJ, et al. Measuring inconsistency in meta-analyses. BMJ. 2003;327(7414): 557-60.

13. Hirshberg B, Parker A, Edelberg H, et al. Safety of saxagliptin: events of special interest in 9156 patients with type 2 diabetes mellitus. Diabetes Metab Res Rev. 2014;30(7):556-69.

14. Janghorbani M, Van Dam RM, Willett WC, et al. Systematic review of type 1 and type 2 diabetes mellitus and risk of fracture. Am J Epidemiol. 2007;166(5):495-505.

15. Josse RG, Majumdar SR, Zheng Y, et al. Sitagliptin and risk of fractures in type 2 diabetes: results from the TECOS trial. Diabetes Obes Metab. 2017;19(1):78-86.

16. Kraenzlin M, Jick H, Meier C, et al. Use of thiazolidinediones and fracture risk. Arch Intern Med. 2008;168(8):820-5.

17. Losada-Grande E, Hawley S, Soldevila B, et al. Insulin use and excess fracture risk in patients with type 2 diabetes: a propensity-matched cohort analysis. Sci Rep. 2017;7(1):3781.

18. Mabilleau G, Mieczkowska A, Irwin N, et al. Optimal bone mechanical and material properties require a functional glucagon-like peptide-1 receptor. J Endocrinol. 2013;219(1):59-68.

19. Mabilleau G, Mieczkowska A, Chappard D. Use of glucagon-like peptide-1 receptor agonists and bone fractures: a meta-analysis of randomized clinical trials. J Diabetes. 2013;6(3):260.

20. Mabilleau G, Perrot R, Mieczkowska A, et al. Glucose-dependent insulinotropic polypeptide (GIP) dose-dependently reduces osteoclast differentiation and resorption. Bone. 2016;91:102-12.

21. Mamza J, Marlin C, Wang C, et al. DPP-4 inhibitor therapy and bone fractures in people with type 2 diabetes: a systematic review and meta-analysis. Diabetes Res Clin Pract. 2016;116:288-98.
22. Mieczkowska A, Irwin N, Flatt PR, et al. Glucosedependent insulinotropic polypeptide (GIP) receptor deletion leads to reduced bone strength and quality. Bone. 2013;56(2):337-42.

23. Monami M, Dicembrini I, Antenore A, et al. Dipeptidyl peptidase- 4 inhibitors and bone fractures: a meta-analysis of randomized clinical trials. Diabetes Care. 2011;34(11):2474-6.

24. Nucheberenguer B, Moreno P, PortalNuñez S, et al. Exendin-4 exerts osteogenic actions in insulin-resistant and type 2 diabetic states. Regul Pept. 2010;159(1):61-6.

25. Palermo A, D'Onofrio L, Eastell R, et al. Oral antidiabetic drugs and fracture risk, cut to the bone: safe or dangerous? A narrative review. Osteoporos Int. $2015 ; 26(8): 2073-89$.

26. Peters JPM, Hooft L, Grolman W, et al. Reporting quality of systematic reviews and meta-analyses of otorhinolaryngologic articles based on the PRISMA statement. PLoS One. 2015;10(8):e0136540.

27. Raz I, Bhatt DL, Hirshberg B, et al. Incidence of pancreatitis and pancreatic cancer in a randomized controlled multicenter trial (SAVOR-TIMI 53) of the dipeptidyl peptidase- 4 inhibitor saxagliptin. Diabetes Care. 2014;37(9):2435-41.

28. Sellmeyer DE, Civitelli R, Hofbauer LC, et al. Skeletal metabolism, fracture risk, and fracture outcomes in type 1 and type 2 diabetes. Diabetes. 2016;65(7):1757-66.

29. Stolk R. Hyperinsulinemia and bone mineral density in an elderly population: the Rotterdam study. Bone. 1996;18(6):545-9.

30. Su B, Sheng H, Zhang M, et al. Risk of bone fractures associated with glucagon-like peptide-1 receptor agonists' treatment: a meta-analysis of randomized controlled trials. Endocrine. 2015;48(1):107-15.

31. Vestergaard P. Discrepancies in bone mineral density and fracture risk in patients with type 1 and type 2 diabetes: a meta-analysis. Osteoporos Int. 2007;18(4):427-44.

32. Yamada C, Yamada Y, Tsukiyama K, et al. The murine glucagon-like peptide-1 receptor is essential for control of bone resorption. Endocrinology. 2008;149(2):574-9.

33. Zhang YS, Weng WY, Xie BC, et al. Glucagon-like peptide-1 receptor agonists and fracture risk: a network meta-analysis of randomized clinical trials. Osteoporos Int. 2018;29(12):2639-44. 